\title{
Ammonium nutrition as a strategy for cadmium mobilisation in the rhizosphere of sunflower
}

Patrizia Zaccheo • Laura Crippa •

Valeria Di Muzio Pasta

Published online: 3 October 2007

(C) Springer Science + Business Media B.V. 2007

Erratum to: Plant Soil Vol 283 pages $43-56$

DOI 10.1007/s11104-005-4791-x

3rd author's name was not correct.

Family name should be Di Muzio Pasta

The online version of the original article can be found at http:// dx.doi.org/10.1007/s11104-005-4791-x

P. Zaccheo $(\bowtie) \cdot$ L. Crippa $\cdot$ V. Di Muzio Pasta

Dipartamento di Produzione Vegetale,

Universita degli Studi di Milano,

Via Celoria 2,

I-20133 Milano, Italy

e-mail: patrizia.zaccheo@unimi.it 